\title{
QUADRATIC MORSE-SMALE VECTOR FIELDS WHICH ARE NOT STRUCTURALLY STABLE
}

\author{
CARMEN CHICONE ${ }^{1}$ AND DOUGLAS S. SHAFER
}

\begin{abstract}
An example is given of a quadratic system in the plane which is Morse-Smale but not structurally stable. Also, it is proved that no such example exists for a quadratic system which is a gradient.
\end{abstract}

1. Introduction. A vector field $X$ on a manifold $M$ is called Morse-Smale if $X$ generates a flow $\varphi_{t}$ such that the nonwandering set $\Omega$ of $\varphi_{t}$ is the union of a finite number of hyperbolic critical points and a finite number of hyperbolic periodic orbits, and the stable and unstable manifolds of the critical points and closed orbits intersect transversally. Given a topology on the space of vector fields, $X$ is called structurally stable if all sufficiently small perturbations of $X$ have the same phase portrait as $X$, up to topological equivalence. If $M$ is compact, a Morse-Smale vector field is structurally stable $[8,9]$, but a Morse-Smale vector field may fail to be structurally stable when $M$ is not compact.

Krych and Nitecki [7] have asked if there is a quadratic system on the plane $R^{2}$ which is Morse-Smale but not structurally stable in the $C^{r}$-Whitney topology [5]. A quadratic system on $R^{2}$ is an autonomous differential equation of form

$$
\dot{x}=P(x, y), \quad \dot{y}=Q(x, y)
$$

where $P$ and $Q$ are polynomials of degree less than or equal to two. In $\$ 2$ we show that such an example is provided by the quadratic system

$$
\dot{x}=2 x y, \quad \dot{y}=2 x y-x^{2}+y^{2}+1 .
$$

In $\$ 3$ we show that the quadratic gradient vector fields which are Morse-Smale are structurally stable in the $C^{r}$-Whitney topology. This strengthens the results in [3] which show that generically, in the coefficient topology, quadratic gradients are Morse-Smale.

Finally, in $\$ 4$ we show that the choice of topology can be crucial for stability by showing that the Morse-Smale quadratic gradient

$$
\dot{x}=-y, \quad \dot{y}=3 y^{2}-x
$$

is not structurally stable in the coefficient topology.

Received by the editors July 16, 1981.

1980 Mathematics Subject Classification. Primary 58F10, 34D30.

Key words and phrases. Morse-Smale, structural stability, quadratic system.

'Research supported by a grant from the Research Council of the Graduate School, University of Missouri. 
We thank Michal Krych and Zbigniew Nitecki for suggesting the problems solved in this paper. We especially wish to thank Michal Krych for helping us find a simpler Dulac function in $\$ 2$.

Before the main body of the paper we should make one last preliminary remark on a technical point. While uniqueness of solutions guarantees that no trajectory of $X$ can tend to a critical point in finite time, by noncompactness of $R^{2}$ it is possible that a trajectory tends to infinity in finite time. If it does so, then the maximum interval of existence of the solution through any point on the trajectory is not all of $R$. In this event, however, we may replace $X$ by the vector field $\tilde{X}=$ $\left(1+\|X\|^{2}\right)^{-1 / 2} \cdot X ;\|\tilde{X}\|$ is uniformly bounded on $R^{2}$ by 1 so its solutions exist on all of $R$. But since $\tilde{X}$ is obtained from $X$ merely by rescaling by a positive function, $\tilde{X}$ has the same integral curves (including restpoints) as $X$, and its critical points are hyperbolic if and only if those of $X$ are. Moreover the map $X \rightarrow \tilde{X}$ is a homeomorphism onto its image (strong $C^{r}$-topology) so, because topological equivalence depends only on the integral curves as they partition the plane and not on their parameterizations, $X$ and $\tilde{X}$ enjoy the same structural stability (or instability). Thus we may safely ignore questions of intervals of existence of solutions and will do so in $\S \S 2$ and 3.

2. A nonstable Morse-Smale quadratic. Consider the quadratic vector field $X$ given by

$$
X(x, y)=2 x y \frac{\partial}{\partial x}+\left(2 x y-x^{2}+y^{2}+1\right) \frac{\partial}{\partial y}
$$

Proposition 2.1. The vector field $X$ is Morse-Smale. There exists a $C^{\infty}$ vector field $Y$ with compact support on $R^{2}$ such that for any $\varepsilon>0, X+\varepsilon Y$ is not topologically equivalent to $X$. In particular, $X$ is not structurally stable in any $C^{r}$-topology.

Proof. The line $L: x=0$ is an invariant set for $X$ and the only critical points in the finite plane are $p=(1,0)$ and $q=(-1,0)$. Moreover, checking the linear part of $X$ at the critical points reveals that $p$ is a hyperbolic clockwise spiral source with eigenvalues $1 \pm \sqrt{3} i$ and $q$ is a hyperbolic counterclockwise spiral sink with eigenvalues $-1 \pm \sqrt{3} i$.

We will show that $X$ has the following properties: (a) the basin of attraction $B^{+}$of the sink $q$ is $\{(x, y): x<0\}$ and the basin $B^{-}$of attraction in reverse time of the source $p$ is $\{(x, y): x>0\}$, (b) there are no periodic orbits, and (c) the nonwandering set $\Omega=\{p, q\}$. These properties imply that $X$ is Morse-Smale and that $B^{+} \cap B^{-}$ $=\varnothing$.

Given (a), (b) and (c), let $B=\left\{(x, y) \mid x^{2}+y^{2}<\frac{1}{4}\right\}$ and construct a $C^{\infty}$ bump function $\rho(x, y)$ such that $0 \leqslant \rho \leqslant 1$ and the support of $\rho$ is contained in $B$. Define $Y=-\rho \partial / \partial x$ and set $Z=X+\varepsilon Y$. Clearly, $Z$ is $C^{r}$ close to $X$ for sufficiently small $\varepsilon>0$ (depending on $r$ ) and $Z$ is equal to $X$ on the complement of $B$. However, for $Z$, it is true that $(0,0) \in B^{+} \cap B^{-}$so that $Z$ is not topologically equivalent to $X$. That is, $X$ is not structurally stable in any $C^{r}$-topology. 
It remains to prove the properties (a), (b), and (c). To show (a) and (b) we identify $R^{2}$ with the set $\{(x, y, z) \mid z=1\} \subseteq R^{3}$ and centrally project onto $S^{2}=\{(x, y, z) \mid$ $\left.x^{2}+y^{2}+z^{2}=1\right\} \subseteq R^{3}$ to obtain a vector field $\tilde{X}$ on the open upper hemisphere, which corresponds to the finite plane. After multiplying $\tilde{X}$ by $z^{2}$ we obtain an analytic extension of $\tilde{X}$ to the equator of $S^{2}$, which corresponds to the line at infinity, thus obtaining a vector field $X_{\infty}$ on the compact upper hemisphere. See [4] for details and explicit formulas for $X_{\infty}$ in terms of the coordinate charts $\left(U_{1}, \psi_{1}\right)$, $\left(U_{2}, \psi_{2}\right)$ and $\left(V_{1}, \chi_{1}\right)$ used below. Note also that the equator of $S^{2}$, denoted $S^{1}$, is always an invariant set for $X_{\infty}$ in this construction.

In the coordinate charts

$$
\left(U_{1}=\{(x, y, z) \mid x>0\} ; \psi_{1}(x, y, z)=\left(\frac{y}{x}, \frac{z}{x}\right)=(s, t)\right)
$$

and

$$
\left(U_{2}=\{(x, y, z) \mid y>0\} ; \psi_{2}(x, y, z)=\left(\frac{x}{y}, \frac{z}{y}\right)=(s, t)\right),
$$

$X_{\infty}$ has the local representations

$$
X_{1}(s, t)=D(s, t)\left\{\left(-s^{2}+t^{2}+2 s-1\right) \partial / \partial s-2 s t \partial / \partial t\right\}
$$

and

$$
X_{2}(s, t)=D(s, t)\left\{s\left(s^{2}-2 s+1-t^{2}\right) \partial / \partial s+t\left(s^{2}-t^{2}-2 s-1\right) \partial / \partial t\right\}
$$

where $D(s, t)^{-1}=\left(1+s^{2}+t^{2}\right)^{1 / 2} \geqslant 1$. Here the line given by $t=0$ corresponds to the line at infinity. In fact, along the line at infinity

$$
X_{1}(s, 0)=D(s, 0)\left(-s^{2}+2 s-1\right) \partial / \partial s
$$

which has a double zero at $(1,0)$, and

$$
X_{2}(s, 0)=D(s, 0)\left(s\left(s^{2}-2 s+1\right)\right) \partial / \partial s
$$

which has a double zero at $(1,0)$ and a single zero at $(0,0)$. After rescaling by $D^{-1}$ the linear part of $X_{2}$ at $(s, t)$ is

$$
\left[\begin{array}{cc}
3 s^{2}-4 s+1 & -2 s t \\
2 s t-2 t & s^{2}-3 t^{2}-2 s-1
\end{array}\right]
$$

so the linear parts at $(0,0)$ and $(1,0)$ are respectively

$$
\left[\begin{array}{cc}
1 & 0 \\
0 & -1
\end{array}\right] \text { and }\left[\begin{array}{cc}
0 & 0 \\
0 & -2
\end{array}\right]
$$

Near $(s, t)=(0,0)$ in $U_{2}, X_{2}$ is a reparametrization of a vector field with a hyperbolic saddle and its phase portrait is as shown in Figure 1. Near $(s, t)=(1,0)$, for $t>0$ (corresponding to the finite plane) the component of $\partial / \partial t$ is negative; hence, no point $(s, t)$ with $t>0$ tends to $(1,0)$ in backward time and the phase portrait is as shown in Figure 2.

If we choose charts corresponding to $V_{1}=\{(x, y, z) \mid x<0\}$ and $V_{2}=\{(x, y, z) \mid$ $y<0\}$ then $X_{\infty}$ has the same representations as in $U_{1}$ and $U_{2}$ except for the scale factor -1 . Hence, the phase portrait of $X_{\infty}$ on the upper hemisphere of $S^{2}$ is as shown in Figure 3. 


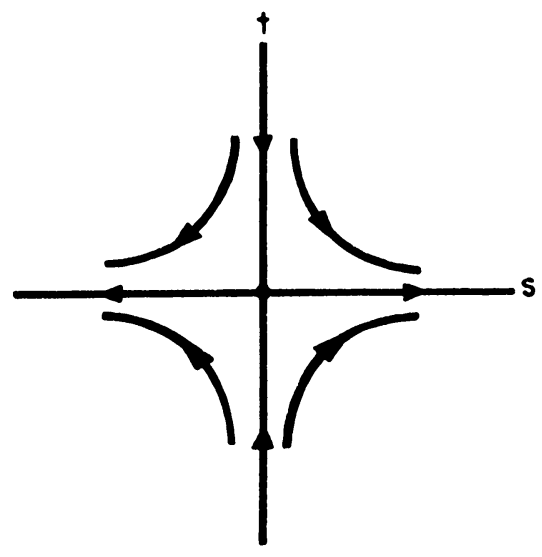

FIGURE 1

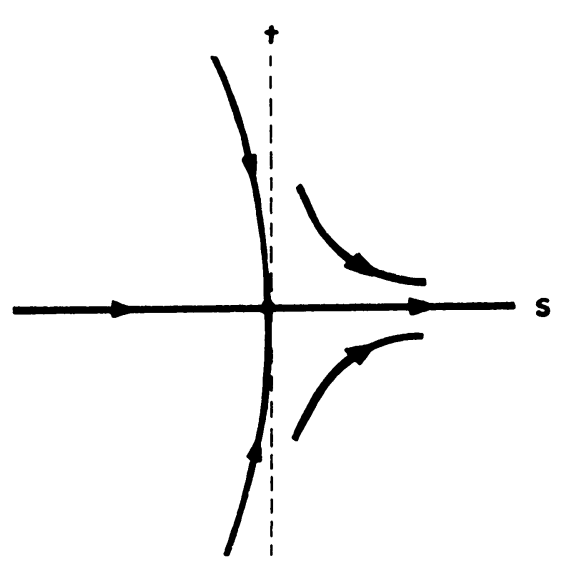

FIGURE 2

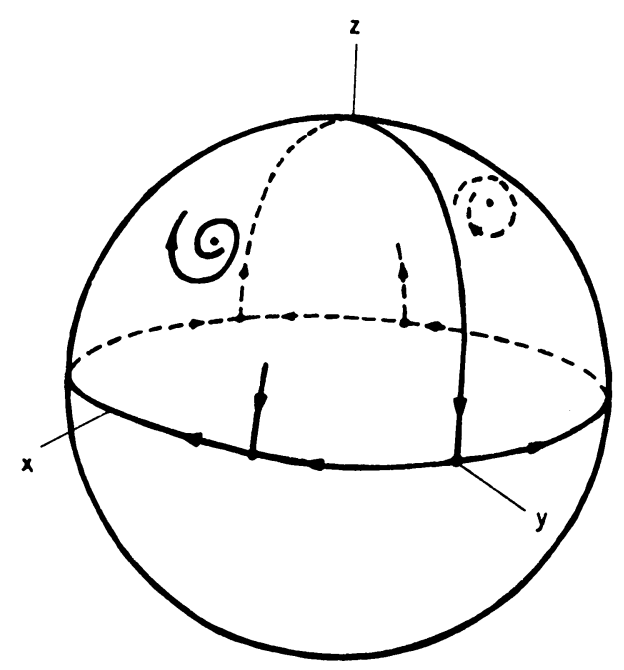

FIGURE 3

To show that there are no periodic orbits, define the Dulac function $B=2 x^{-2}$ and compute the divergence of the vector field $B X$. We obtain $\operatorname{div}(B X)=4 x^{-1}$; hence by Dulac's criterion [1, p. 205] there is no periodic orbit contained in the open right or left half plane. Since the line given by $x=0$ is invariant, no periodic orbit can intersect both half planes and it follows that there are no periodic orbits. Projecting the phase portrait from the closed upper hemisphere onto the closed unit disk of the $x y$-plane we obtain the phase portrait shown in Figure 4.

This establishes (a) and (b). But in view of the obvious fact that no point on the line $L: x=0$ is in $\Omega$, we have $\Omega=\{p, q\}$, which is (c). Q.E.D. 


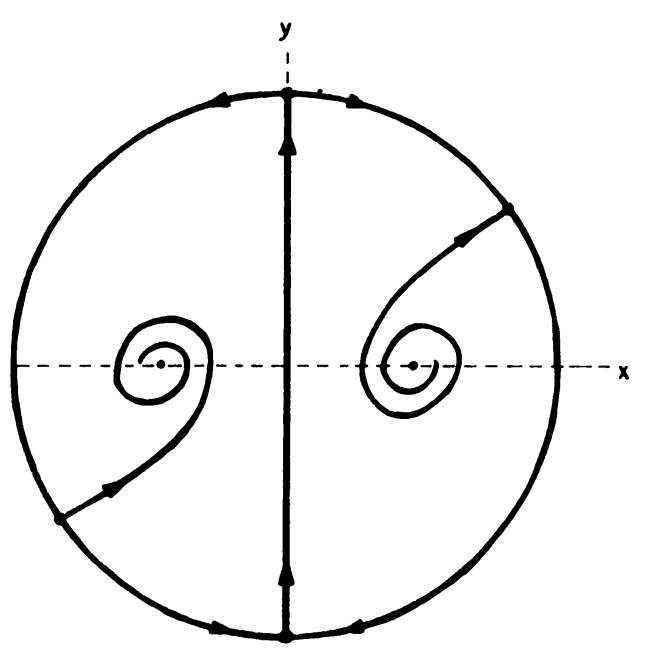

Figure 4

3. Quadratic gradients. In this section we study quadratic systems which are gradients of cubic potential functions. Let $H$ denote the general cubic in two variables with real coefficients given by

$$
H(x, y)=a x^{3}+b x^{2} y+c x y^{2}+d y^{3}+e x^{2}+f x y+g y^{2}+h x+k y+m .
$$

Recall, that the gradient $\nabla H$ of $H$ is the quadratic vector field

$$
\nabla H=\frac{\partial H}{\partial x} \frac{\partial}{\partial x}+\frac{\partial H}{\partial y} \frac{\partial}{\partial y}
$$

and that the Hamiltonian vector field $J d H$ is the quadratic vector field

$$
J d H=\frac{\partial H}{\partial y} \frac{\partial}{\partial x}-\frac{\partial H}{\partial x} \frac{\partial}{\partial y} \text {. }
$$

Let $\eta_{t}$ denote the flow of $\nabla H$.

In this section we prove

THEOREM 3.1. If $\nabla H$ is Morse-Smale, then $\nabla H$ is structurally stable in the $C^{r}$-Whitney topology.

The proof of 3.1 will require the theorem of Kotus, Krych and Nitecki which we now explain.

Definition 3.2. A saddle at infinity for a planar vector field $X$ generating a flow $\varphi_{t}$ is a pair of distinct orbits $\left\{\varphi_{t}(p)\right\}$ and $\left\{\varphi_{-t}(q)\right\}$ such that (i) $\lim _{t \rightarrow \infty} \varphi_{t}(p)=\infty$ and $\lim _{t \rightarrow \infty} \varphi_{-t}(q)=\infty$, and (ii) there exist sequences $p_{n} \rightarrow p, t_{n} \rightarrow \infty$ such that $\lim _{n \rightarrow \infty} \varphi_{t_{n}}\left(p_{n}\right)=q$, or there exist sequences $q_{n} \rightarrow q, t_{n} \rightarrow \infty$ such that $\lim _{n \rightarrow \infty} \varphi_{-t_{n}}\left(q_{n}\right)=p$. Moreover, in the first case of (ii) $\left\{\varphi_{t}(p)\right\}$ is in the stable manifold of the saddle at infinity and in the second case $\left\{\varphi_{1}(q)\right\}$ is in the unstable manifold of the saddle at infinity. 
Let $W_{s}$ denote the union of all stable manifolds of all finite and infinite saddles of $X$ and let $W_{u}$ denote the union of all unstable manifolds of all finite and infinite saddles of $X$. Recall that a minimal set is a nonempty compact invariant set with no proper compact invariant subset. Also, we say that an orbit oscillates if its $\omega$-limit set is a noncompact subset of $R^{2}$.

THEOREM 3.3 (KOTUS, KRYCH AND NITECKI [6]). The vector field $X$ is structurally stable in the $C^{r}$-Whitney topology if and only if (i) all critical points and periodic orbits are hyperbolic, (ii) every minimal set is either a periodic orbit or a fixed point, (iii) there are no oscillating orbits and (iv) closure $\left(W_{s}\right) \cap$ closure $\left(W_{u}\right)$ consists of finite saddle points only.

DEFINITION 3.4. A saddle connection at infinity is an orbit which is both the stable manifold for a saddle at infinity and the unstable manifold for a saddle at infinity.

Proposition 3.5. If $\nabla H$ is Morse-Smale but not structurally stable, then $\nabla H$ has a saddle connection at infinity.

Proof. Since $\nabla H$ is a Morse-Smale gradient vector field, there are no minimal sets which are not fixed points, there are no oscillating orbits, and all critical points are hyperbolic. Since $\nabla H$ is a quadratic system there are at most a finite number of stable (unstable) manifolds of saddle points in the finite plane and at infinity. Hence, by Theorem 3.3 there must be a saddle connection, i.e., an orbit in $W_{s} \cap W_{u}$. But, there are no finite saddle connections since $\nabla H$ is Morse-Smale. Q.E.D.

Definition 3.6. A Reeb component of a flow in the plane is a region $R$ in the plane foliated by orbits of the flow such that the flow on $R$ is topologically equivalent to the flow of the quadratic vector field $\left(x^{2}-1\right) \partial / \partial x+x \partial / \partial y$ in the strip $\{(x, y) \mid-1 \leqslant x \leqslant 1\}$.

Question. A quadratic system (which is not a gradient) can have a Reeb component (3.6). Can a quadratic system have two Reeb components?

LEMMA 3.7. Critical points of $J d H_{\infty}$ along the equator correspond to lines in $R^{2}$ along which the cubic polynomial

$$
H_{3}(x, y)=a x^{3}+b x^{2} y+c x y^{2}+d y^{3}
$$

(the cubic terms of $H$ ) is zero.

Proof. In the coordinate chart $\left(U_{1}, \psi_{1}\right)$ on $S^{2} s$ the extended central projection $J d H_{\infty}$ of $J d H$ is

$$
J d H_{\infty}(s, t)=D(s, t)\left[-3 a-3 b s-3 c s^{2}-3 d s^{3}+\cdots, \cdots\right],
$$

where the omitted terms all contain $t$ as a factor. Thus along $\{t=0\}$, corresponding to the equator, $J d H_{\infty}(s, 0)=D(s, 0)\left[-3 H_{3}\left(1, s^{3}\right), 0\right]$, and $J d H_{\infty}$ vanishes at $s=s_{0}$ if and only if $H_{3}$ vanishes along the line $y=s_{0} x$. Q.E.D.

The next lemma depends on the fact that $\nabla H$ is the gradient of a cubic potential. The conclusions of the lemma are false for a general quadratic system, and it is this fact which allowed the construction of the example in $\$ 2$. 
LEMma 3.8. Let $\left\{\eta_{t}(p)\right\}$ and $\left\{\eta_{-t}(q)\right\}$ be a saddle at infinity (Definition 3.2) for $\nabla H$. Then the corresponding orbits of $\nabla H_{\infty}$ tend to the same point on the equator $S^{1}$, and this point is a critical point of both $\nabla H_{\infty}$ and $J d H_{\infty}$. Moreover it is a double zero of $\nabla H_{\infty} \mid S^{1}$.

Proof. Since $H$ is continuous and strictly increases on orbits of $\nabla H$, we have that

$$
H(p)<H\left(\eta_{t}(p)\right)<H\left(\eta_{-t}(q)\right)<H(q)
$$

for all $t>0$. But then the function $t \mapsto H\left(\eta_{t}(p)\right)$ is monotone increasing and bounded above, hence has a finite limit. Writing $\eta_{t}(p)=(r(t) \cos \theta(t), r(t) \sin \theta(t))$ and $H(x, y)=H_{3}(x, y)+H_{2}(x, y)+H_{1}(x, y)+m$, where $H_{i}(x, y)$ is a polynomial of degree $i, i=1,2,3$, then

$$
H\left(\eta_{t}(p)\right)=r(t)^{3}\left[\sum_{i=1}^{3} r(t)^{i-3} H_{i}(\cos \theta(t), \sin \theta(t))+r(t)^{-3} \cdot m\right] .
$$

Clearly $\lim _{t \rightarrow+\infty} H\left(\eta_{t}(p)\right)=\lim _{t \rightarrow+\infty} r(t)^{3} H_{3}(\cos \theta(t)$, $\sin \theta(t))$. Since $r(t)$ grows without bound, $H_{3}(\cos \theta(t), \sin \theta(t))$ tends to zero. But $H_{3}(x, y)$ is a homogeneous cubic polynomial function, hence $\theta(t)$ tends to a limit $\theta_{0} \in[0,2 \pi)$, which is the angle made between the horizontal and a line $l$ along which $H_{3}$ vanishes. Then the central projection of $\eta_{t}(p)$ onto $S^{2}$ tends to a point $A$ on the equator, which by the Poincaré-Bendixson Theorem applied to $S^{2}$ is a critical point of $\nabla H_{\infty}$. But by Lemma 3.7 and the comment immediately above, $A$ is a critical point of $J d H_{\infty}$ as well. The same considerations apply to $\eta_{-t}(q)$ to give a critical point $B$ of $\nabla H_{\infty}$ and $J d H_{\infty}$, on the equator of $S^{2}$, to which $\eta_{-t}(q)$ tends as $t$ tends to infinity.

In the coordinate chart $\left(U_{1}, \psi_{1}\right)$ on $S^{2}, \nabla H_{\infty}$ is

$$
\nabla H_{\infty}(s, t)=D(s, t)[b+(2 c-3 a) s+f t+\cdots,-3 a t+\cdots]
$$

where the omitted terms are of second order in $s$ and $t$. By a rotation of the coordinates in $R^{2}$ we may place $A$ at $(s, t)=(0,0)$. Then the expressions in coordinates in $U_{1}$ for $J d H_{\infty}$ (proof of Lemma 3.7) and $\nabla H_{\infty}$ (above) show that $a=b=0$, so the linear part of $\nabla H_{\infty}$ at $A$ is

$$
\left(\begin{array}{cc}
2 c & f \\
0 & 0
\end{array}\right)
$$

If $c$ were nonzero $\nabla H_{\infty}$ would have a node, saddle-node, or saddle with two tangent directions at $(s, t)=(0,0)$, which is impossible since $\{t=0\}$ (the equator) is invariant and there are two separatrices (the projections of $\eta_{t}(p)$ and $\left.\eta_{-t}(q)\right)$ in the upper half-plane $\{t>0\}$ (the upper hemisphere). Thus $c=0$ and $\nabla H_{\infty}$ has a double zero at $A$.

But in these coordinates $\nabla H_{\infty} \mid S^{1}$ is a cubic polynomial in $s$ and $t$ times the nonvanishing factor $D(s, t)$. Since it similarly has a double root at $B$ as well, it must be the case that $A=B$. Q.E.D.

REMARK 3.9. By the proof of 3.8, $\nabla H_{\infty} \mid S^{1}$ has a double zero at a point $A$ (and its antipodal point $A^{\prime}$ ), hence a single zero at a second point $C$ (and its antipodal point $\left.C^{\prime}\right)$. Placing $A$ at $(1,0,0), C$ is easily found to be at $(0,1,0)((s, t)=(0,0)$ in the $\left.\operatorname{chart}\left(U_{2}, \psi_{2}\right)\right)$. 
Proof of Theorem 3.1. Let $H$ be such that $\nabla H$ is Morse-Smale. By Proposition 3.5 $\nabla H$ is not structurally stable only if there is a saddle connection at infinity. Hence, we assume there is a point $p$ on such a saddle connection and obtain a contradiction.

Since $p$ is in the stable manifold of a saddle at infinity, the projection of $\eta_{t}(p)$ onto $S^{2}$ tends to a critical point $A$ on the equator $S^{1}$, which by a rotation may be placed at $(1,0,0)$. By Lemma 3.8 and Remark 3.9, $\nabla H_{\infty} \mid S^{1}$ has double zeros at $A$ : $(1,0,0)$ and $A^{\prime}:(-1,0,0)$ and single zeros at $C:(0,1,0)$ and $C^{\prime}:(0,-1,0)$, so we have in these coordinates (in $R^{2}$ ) that $a=b=c=0$.

Now $f \neq 0$, otherwise in the chart $\left(U_{1}, \psi_{1}\right)$

$$
\nabla H_{\infty}(s, t)=D(s, t)\left[*,-t^{2}(2 e+h t)\right]
$$

We observe that the second component has constant sign either in the positive half plane $\{t>0\}$, or in the strip $\{(s, t): 0<t<-2 e / h\}$. In either case $\nabla H_{\infty}$ cannot have a hyperbolic sector contained in the positive half plane, which corresponds to the finite plane.
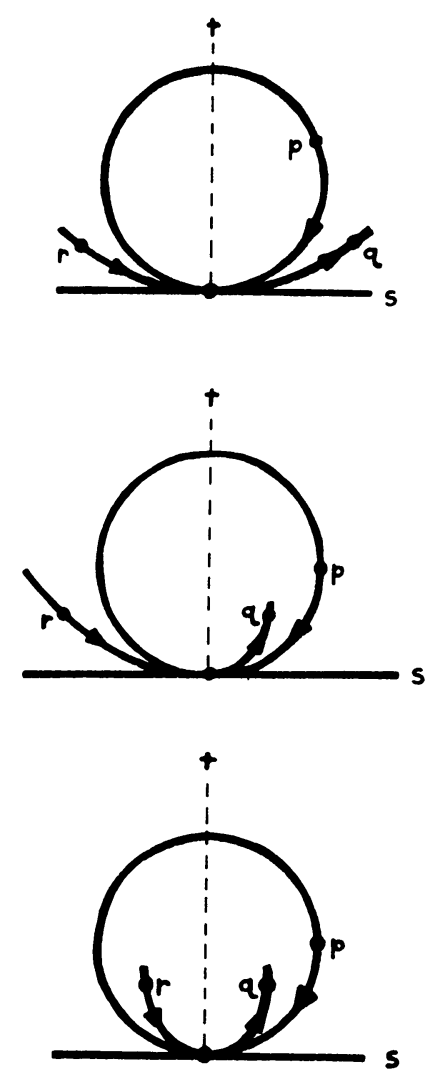
If $p$ is also in the unstable manifold of a saddle at infinity, the projection of $\eta_{-t}(p)$ onto $S^{2}$ also tends to a critical point of $\nabla H_{\infty}$ on $S^{1}$. By Lemma 3.8 it is a double zero of $\nabla H_{\infty} \mid S^{1}$, hence is either $A$ or $A^{\prime}$. But the fact that $f \neq 0$ implies that $\nabla H_{\infty}$ has nonzero linear part at $A$ (see the proof of 3.8 ), so we may consult the catalogue of all possible phase portraits [1, Theorems 66 and 67, pp. 357, 362] to exclude $A$ and $A^{\prime}$ in turn, giving the contradiction.

To exclude $A$, let $q$ be such that $\left\{\eta_{t}(p)\right\}$ and $\left\{\eta_{-t}(q)\right\}$ form a saddle at infinity ( $p$ in the saddle manifold) and $r$ such that $\left\{\eta_{t}(r)\right\}$ and $\left\{\eta_{-t}(p)\right\}$ form a saddle at infinity ( $p$ in the unstable manifold). The projections of the trajectories through $p, q$, and $r$ give rise to hyperbolic sectors of $\nabla H_{\infty}$ at $A$, while the invariance of the equator gives other hyperbolic, elliptic, or parabolic sectors, depending on the direction of flow along the equator and whether the orbit through $p$ bounds $q$ or $r$. (See Figure 5, shown in coordinates $(s, t)$ in $U_{1} ;\{t=0\}$ is the line at infinity, $\{t>0\}$ is the finite plane.)

In every case the phase portrait does not appear in the catalogue, so $A$ is excluded.

To exclude $A^{\prime}$, choose about $A^{\prime}$ the chart $\left(V_{1}=\{(x, y, z) \mid x<0\}\right.$; $\left.\chi_{1}(x, y, z)=\left(\frac{y}{x}, \frac{z}{x}\right)=(s, t)\right)$. Then the coordinate expression for $\nabla H_{\infty}$ in $V_{1}$ is $(-1)$ times that in $U_{1}$. Thus the behavior of $\nabla H_{\infty}$ in the upper hemisphere (corresponding to the finite plane) near $A^{\prime}$ is exactly that of $\nabla H_{\infty}$ in the lower hemisphere near $A$. But, again, inspection of possible phase portraits shows that $\nabla H_{\infty}$ cannot have the equator invariant, $A$ a double point for $\nabla H_{\infty} \mid S^{1}$, and two separatrices in each hemisphere, so $A^{\prime}$ is excluded. Q.E.D.

In the proof of 3.1 we made decisive use of the catalogue of possible phase portraits for degenerate singularities with nonzero linear part. This does not depend on the fact that the gradient was a quadratic. Hence, it is natural to ask if the analogue of 3.1 holds for gradients of higher degree.

Conjecture 3.10. If $H$ is quartic and $H$ is Morse-Smale, then $\nabla H$ is structurally stable.

Conjecture 3.11. There exists a quintic potential $H$ such that $\nabla H$ is Morse-Smale but not structurally stable.

4. A perturbation in the coefficient topology. In this section we show that the gradient of the cubic potential $H(x, y)=y\left(y^{2}-x\right)$ is Morse-Smale, but is not structurally stable in the coefficient topology. In fact, we show that it is not structurally stable in the coefficient topology of the space of cubic potentials. Of course, the gradient is structurally stable in the $C^{r}$-Whitney topology.

Observe that the gradient of $H$ is the vector field $X$ given by

$$
X=-y \partial / \partial x+\left(3 y^{2}-x\right) \partial / \partial y
$$

that $X$ has a hyperbolic saddle point at the origin and that $X$ has no other critical points in $R^{2}$. It follows that $X$ is Morse-Smale.

We consider for $\varepsilon \geqslant 0$, the family of potentials

$$
H_{\varepsilon}(x, y)=(\varepsilon x+y)\left(y^{2}-x\right)
$$


and the corresponding gradients

$$
X_{\varepsilon}(x, y)=\left(\varepsilon y^{2}-2 \varepsilon x-y\right) \partial / \partial x+\left(2 \varepsilon x y+3 y^{2}-x\right) \partial / \partial y .
$$

Clearly, $X$ has coefficients $\varepsilon$-close to $X$ so that $X$ can be made arbitrarily close to $X$ in the coefficient topology. However, we note that $X_{\varepsilon}$ is not close to $X$ in the $C^{r}$-Whitney topology because the perturbation

$$
\left(\varepsilon y^{2}-2 \varepsilon x\right) \partial / \partial x+2 \varepsilon x y \partial / \partial y
$$

is large near infinity. Since $X_{\varepsilon}$ has two critical points $\left((0,0)\right.$ and $\left.\left(1 / \varepsilon^{2},-1 / \varepsilon\right)\right), X_{\varepsilon}$ is not topologically equivalent to $X$ for any $\varepsilon>0$.

\section{References}

1. A. Andronov et al., Qualitative theory of second-order dynamic systems, Wiley, New York, 1973.

2. __ Theory of bifurcations of dynamic systems on the plane, Wiley, New York, 1973.

3. C. Chicone, Quadratic gradients on the plane are generically Morse-Smale, J. Differential Equations 33 (1979), 159-166.

4. E. Gonzalez Velasco, Generic properties of polynomial vector fields at infinity, Trans. Amer. Math. Soc. 143 (1969), 201-222.

5. M. Hirsch, Differential topology, Springer-Verlag, New York, 1976.

6. J. Kotus, M. Krych and Z. Nitecki, Global structural stability of flows on open surfaces, Mem. Amer. Math. Soc. (to appear).

7. M. Krych and Z. Nitecki, Private Communication.

8. J. Palis and S. Smale, Structural stability theorems, Global Analysis, Part I (S. S. Chern and S. Smale, Eds.), Proc. Sympos. Pure Math., vol. 14, Amer. Math. Soc., Providence, R. I., 1970, pp. 223-231.

9. M. Piexoto, Structural stability on two dimensional manifolds, Topology 1 (1962), 101-120.

Department of Mathematics, University of Missouri, Columbia, Missouri 65211

Department of Mathematics, University of North Carolina, Charlotte, North Carolina 28223 